\title{
Mechanisms controlling the volume of pleural fluid and extravascular lung water
}

\author{
G. Miserocchi
}

ABSTRACT: Pleural and interstitial lung fluid volumes are strictly controlled and maintained at the minimum thanks to the ability of lymphatics to match the increase in filtration rate. In the pleural cavity, fluid accumulation is easily accommodated by retraction of lung and chest wall (high compliance of the pleural space); the increase of lymph flow per unit increase in pleural fluid volume is high due to the great extension of the parietal lymphatic. However, for the lung interstitium, the increase in lymph flow to match increased filtration does not need to be so great. In fact, increased filtration only causes a minor increase in extravascular water volume $(<10 \%)$ due to a marked increase in interstitial pulmonary pressure (low compliance of the extracellular matrix) which, in turn, buffers further filtration. Accordingly, a less extended lymphatic network is needed. The efficiency of lymphatic control is achieved through a high lymphatic conductance in the pleural fluid and through a low interstitial compliance for the lung interstitium. Fluid volume in both compartments is so strictly controlled that it is difficult to detect initial deviations from the physiological state; thus, a great physiological advantage turns to be a disadvantage on a clinical basis as it prevents an early diagnosis of developing disease.

KEYWORDS: Extracellular matrix, lung oedema, lymphatics, microvascular permeability, pleural effusion, protein reflection coefficient

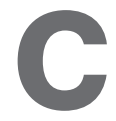
ontrol of extravascular water differs among organs and body compartments reflecting specific functional conditions. In some regions, the control of extravascular water volume is quite loose, such as in the muscles or liver, while in other compartments it is very tight and tuned so as to keep the volume at a minimum. This is the case of the pleural cavity and of the lung interstitial compartment. In both cases, this setting has important functional correlates: for the pleural space, a minimum volume of fluid ensures the maximum intrathoracic expansion of the lung; for the air-blood barrier, a minimum volume of interstitial water assures the maximum surface/ thickness ratio to optimise gas diffusion. The aim of this article is to describe the mechanisms controlling the volume of water in the pleural space and in the lung interstitium. In fact, control mechanisms have been somehow overlooked, likely due to the complexity of some aspects of liquid turnover and the difficulty to measure key variables. As we shall see, powerful control mechanisms exist, both for pleural liquid and for lung interstitial space, which are strong enough to offset transient conditions of increase in water content.

\section{INTERACTION BETWEEN LUNG MECHANICS AND EXTRAVASCULAR FLUID DYNAMICS}

A key variable to discuss lung extravascular fluid dynamics is the pressure at which the extravascular fluid is maintained as it reflects the interaction between the pressures responsible for fluid exchanges, as well as those generated in the surrounding environment which, in turn, relate to lung mechanics. All pressures involved display a gravity dependent distribution; in this article we will ignore this feature and, for simplicity, we will refer to average values at $\sim 50 \%$ lung height.

Pleural fluid is exposed to lung recoil pressure ( $\sim 5 \mathrm{cmH}_{2} \mathrm{O}$ at the functional residual capacity) which is balanced by an equal and opposite pressure exerted by the chest wall (fig. 1a). When seen from the pleural side, this pressure is commonly referred to as pleural surface pressure (in this case a negative sign is given, thus $-5 \mathrm{cmH}_{2} \mathrm{O}$ ). Yet, the pressure of the pleural liquid (Pliq) does not simply reflect pleural surface pressure but also reflects the dynamics of fluid

\section{CORRESPONDENCE}

G. Miserocchi

Dipartimento di Medicina

Sperimentale, Facoltà di Medicina e

Chirurgia

Università di Milano-Bicocca

Via Cadore 48

Monza

Italy.

E-mail:

giuseppe.miserocchi@unimib.it

Received:

May 212009

Accepted after revision:

June 032009

PROVENANCE

Submitted article, peer reviewed.
European Respiratory Review Print ISSN 0905-9180 Online ISSN 1600-0617 
a)

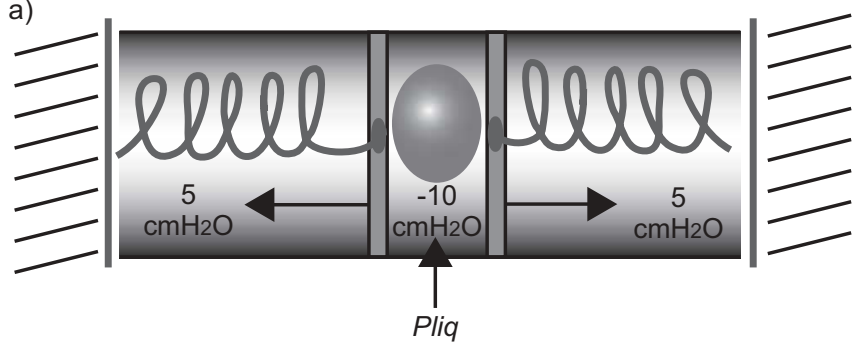

b)

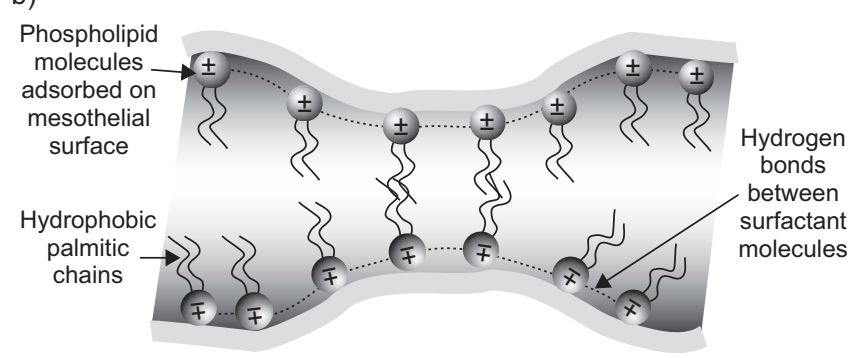

FIGURE 1. a) At functional residual capacity, lung and chest walls exert on each other a recoil pressure equal in module but opposite in sign. Mechanical coupling is assured by pleural liquid pressure (Pliq) that is more negative than the recoil pressure of the structures. As a consequence, the visceral and parietal pleura push one against the other (as suggested by the solid deformed body). b) Actual touching between opposing pleurae does not occur because of repulsive forces between several layers of phospholipids adsorbed on mesothelial surfaces carrying charges of the same sign.

turnover across the pleural membranes and, in fact, it is more sub-atmospheric averaging $-10 \mathrm{cmH}_{2} \mathrm{O}$ [1]. Accordingly, the visceral and parietal pleura are actually pushing one against the other, as suggested by the solid deformed body. In fact, this body does not exist as such because, as shown in figure $1 \mathrm{~b}$, pleural surfaces go very close but actually do not touch each other due to reciprocal repulsive forces of charges of the same sign (negative) carried by polar phospholipids adsorbed (actually stratified on several layers) on the opposing pleural surfaces. This arrangement provides a very efficient lubrication system [2]. Figure 2 shows that the pulmonary interstitial pressure $(\mathrm{Pi})$ in the septal region is also approximately $-10 \mathrm{cmH}_{2} \mathrm{O}$ [3] and reflects a complex balance between at least three sets of forces: 1) those governing microvascular-interstitial fluid exchanges; 2) elastic lung recoil forces; and 3) surface forces at the air-tissue interface (the ortogonal component of surface tension is shown in figure 2). The existence of negative pressure has clear mechanical implications: in the pleural cavity the lungs are kept well adherent to the chest wall, and in the lung the endothelium of the capillary wall is tightly glued to the epithelial wall.

\section{THE GENERAL MODEL FOR CONTROL OF EXTRAVASCULAR WATER}

Fluid filtration across biological barriers (e.g. capillary endothelium and pleural mesothelium) is defined by the revisited Starling equation.

$$
J f=K f[(P c-P e v)-\sigma(\pi c-\pi \mathrm{ev})]
$$

Where $K f$ is the filtration coefficient, $P$ and $\pi$ refer to hydraulic and colloid osmotic pressures, respectively, $c$ and $e v$ refer to

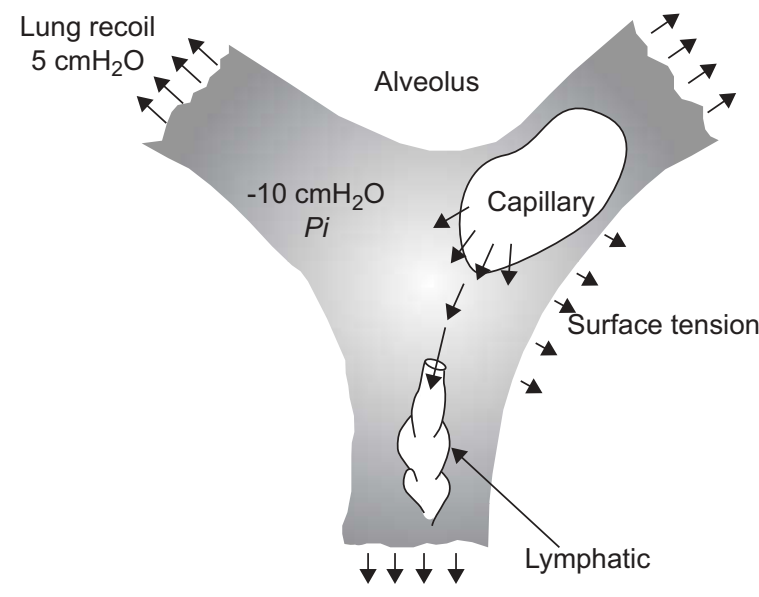

FIGURE 2. Simple schematic diagram of a lung septal region depicting the pressures acting on the unit. Also in this case interstitial pressure $(P i)$ is more negative than that generated by lung recoil pressure.

capillary and extravascular compartments, respectively, and $\sigma$ is the protein reflection coefficient $[4,5]$. Putting the overall pressure balance in square brackets equal to $P f$, the Starling equation simply reads as:

$$
J f=K f \times P f
$$

The filtration coefficient $K f$ is equal to the product $L p \times S$, where $L p$ is the water hydraulic permeability and $S$ is the total surface available for filtration. $L p$ mostly reflects the distribution of small pores of the order of a 5-nm opening on the membrane barrier along the paracellular route, with an average density of $30 \cdot \mu^{-2}$, through which most of the water flows.

Proteins also move across barriers, dragged only by water through a pore system that allows their passage. The protein reflection coefficient defines how easy it is for a given protein of radius $a$ to cross a barrier with pores of radius $R$ and is defined as $\sigma=(1-\varnothing)^{2}$, being $\varnothing=\left(1-\frac{a}{R}\right)^{2}$. The value of $\sigma$ is 1 for $a=R$, and it decreases to approach 0 for $a<<R$. Pores allowing protein transport are in the range of 30-50 nm, with an average density as low as $0.006 \cdot \mu \mathrm{m}^{-2}$, accordingly they provide a minor share of water transport $[4,5]$.

A sensitive index of the reflection coefficient may be estimated from the extravascular space/plasma protein concentration ratio $\frac{P e v}{P p l}$ under conditions of increased microvascular filtration, such as when pleural effusion or lung oedema develop. In such conditions, the simple relationship $\sigma \approx 1-\frac{P e v}{P p l}$ holds true.

When protein sieving is high, $\frac{P e v}{P p l}$ is low and $\sigma \approx 1$ while, for progressively increasing damage of the barriers, proteins are allowed to reach the extravascular compartment so that $\frac{P e v}{P p l}$ increases and, correspondingly, $\sigma$ decreases.

Lymphatic drainage of the interstitial fluid is defined as follows $[6,7]$.

$$
J l=K l(\text { Plabs - Pev) }
$$


Where $K l$ is the conductance of the initial lymphatics, and Plabs and $\mathrm{Pev}$ are the absorption pressure of the lymphatic pump and the pressure in the extravascular compartment, respectively. Plabs has been estimated to be rather sub-atmospheric, attaining approximately -10 to $-15 \mathrm{cmH}_{2} \mathrm{O}$; thus, a good way to think of lymphatics is to compare them to a vacuum cleaner [6, 7]. The lymphatic conductance $K l$ is a coefficient proportional to the extension of the lymphatic network. Note that the difference Plabs - Pev has a negative value, thus indicating that lymph flow provides on outflow from the extravascular compartment.

Although pressure values in equations 1 and 2 may vary among body compartments, the majority of the experimental evidence supports a fluid exchange model, as presented in figure 3 , which is valid in most of the body compartments; namely, Starling dependent filtration is balanced by lymphatic drainage [4-9]. This model is based on modern measurements of extravascular hydraulic and colloid osmotic pressure values from equation 1 and includes the terms relative to the extravascular space (that were clearly not available at the time of the Starling equation), as well as the coefficient of filtration and the reflection coefficient. Therefore, under steady state condition one has $J f+J l \sim 0$.

As we are dealing with pleural fluid and lung interstitial fluid, $P e v$ will actually refer either to Pliq or to Pi.

\section{GENERAL CONCEPTS OF CONTROL}

A control mechanism implies that the controlled variable is maintained around a set point, in our case a minimal value for the steady state volume of pleural and lung interstitial fluid. The relationship between volume and pressure of the extravascular fluid ( $\mathrm{Pev}$ ) is univocally determined by knowing the compliance $(C)$ of the extravascular compartment (either the pleural or the lung interstitial space).

Compliance can be experimentally determined by measuring the increase in pressure associated to the increase in water volume in the compartment, i.e.

$$
\mathrm{C}=\frac{\Delta V}{\Delta P e v}
$$

The average compliance of the pleural space in humans is $\sim 70 \mathrm{~mL} \cdot \mathrm{cmH}_{2} \mathrm{O}^{-1}$ [7], while that of the lung interstitium is $\sim 0.4 \mathrm{~mL} \cdot \mathrm{cmH}_{2} \mathrm{O}^{-1} \times 100 \mathrm{~g}$ wet weight [9]. As such, these values do not allow a direct comparison between the two compartments. To overcome this difficulty, one should

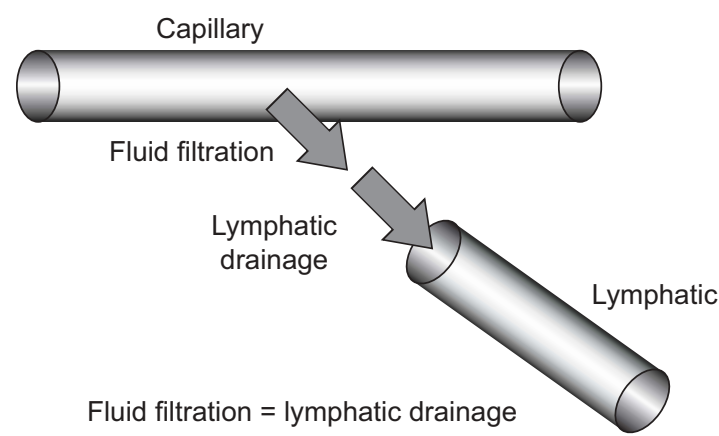

FIGURE 3. Model of control of pleural fluid and lung interstitial fluid volume based on the balance between microvascular filtration and lymphatic absorption. introduce the concept of "specific compliance" $\left(C^{\prime}\right)$, by expressing $\Delta V$ as a change relative to the steady state volume of liquid $\left(V_{0}\right)$ in either compartment, namely,

$$
\Delta V^{\prime}=\frac{\Delta V}{\Delta V_{0}} \text { so that } C^{\prime}=\frac{\Delta V^{\prime}}{\Delta P e v}
$$

For the pleural space, specific compliance is $\sim 10 \cdot \mathrm{cmH}_{2} \mathrm{O}^{-1}$, a very high value because pleural fluid accumulation is easily accommodated by spontaneous retraction of lung and chest wall (Pliq increases by $1 \mathrm{cmH}_{2} \mathrm{O}$ for a 10-times increase in pleural liquid volume). For the lung interstitial space, specific compliance is much lower, $\sim 0.01 \cdot \mathrm{cm} \mathrm{H}_{2} \mathrm{O}^{-1}$.

The control mechanism resides in the ability of lymphatics to increase the draining flow in response to an increase in filtration rate. Considering equation 2, whenever filtration increases, Pev becomes less negative (due to volume accumulation) so that the driving pressure for lymphatics (Plabs - Pev) also increases. The modulation of lymph flow is remarkable because the difference Plabs - Pev is minimal under steady state conditions (Pev is very close to Plabs), and it can increase by $\sim 20$ times.

To appreciate how compliance is affecting the lymph flow response one could differentiate equation 2 with respect to $P e v$, assuming Plabs and $K l$ are constant, to yield

$$
\partial J l=K l \cdot \partial P e v \text { and } \Delta J l=K l \cdot \Delta P e v
$$

from which:

$$
\frac{\Delta J l}{\Delta P e v}=K l
$$

The ratio $\frac{\Delta J l}{\Delta P e v}$ defines the "gain" of the lymphatic control, which is the increase in lymph flow for unit increase in liquid pressure (either Pliq or Pi). As long as the functional design of the lymphatic network is able to face an increased filtration, the ratio $\frac{\Delta J l}{\Delta P e v}$ is exactly equal to the lymphatic conductance $K l$ in either compartment.

Furthermore, from equation 3 one has $\Delta P e v=\frac{\Delta V^{\prime}}{C^{\prime}}$, and substituting into equation 4 :

$$
\frac{K l}{C^{\prime}}=\frac{\Delta J l}{\Delta V}
$$

$\frac{K l}{C^{\prime}}$ defines the "operational features" of the lymphatic control as it includes the two important characteristics of the functional design, namely lymphatic conductance and specific compliance. $\frac{\Delta J}{\Delta V}$ can be regarded as "efficiency" of lymphatic control: the higher this ratio, the smaller the increase in volume of the pleural or lung interstitial fluid after lymphatic flow has adapted to match the increase in filtration rate.

\section{PLEURAL FLUID}

\section{Steady state pleural fluid dynamics}

Pleural fluid turnover averages $0.2 \mathrm{~mL} \cdot \mathrm{kg}^{-1} \cdot \mathrm{h}^{-1}$ [8], which assures complete fluid renewal in $\sim 1 \mathrm{~h}$. Fluid filters through the parietal pleura while drainage is mostly provided $[7,8]$ by the lymphatic stomata of the parietal pleura, which are opening $0.3-40 \mu \mathrm{m}$ in diameter (either single or in cluster) and directly connecting the pleural space with the parietal lymphatic draining system [10]. The extension of the lymphatic 


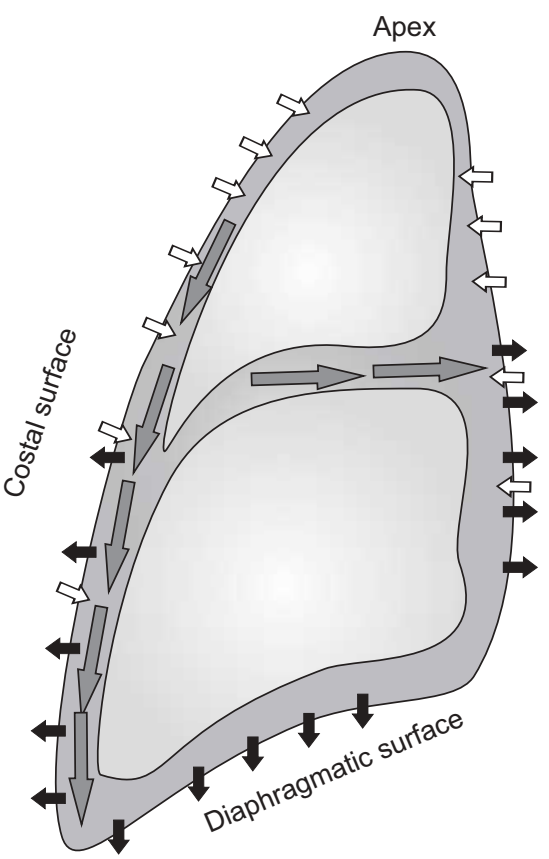

3
0
0
00
01
05
0
0
0
0
0
00
00
0

FIGURE 4. Polarisation of filtration/drainage processes in the pleural cavity and of intrapleural fluxes. White arrow: filtration; black arrow: lymphatic drainage; grey arrow: intrapleural fluxes.

network is mostly developed on the diaphragmatic surface where the density of stomata reaches the considerable value of $8,000 \cdot \mathrm{cm}^{-2}$, and on the mediastinal side where the density of stomata is so high that one speaks of a "cribriform membrane"; the mesothelial openings connect to an extended network of submesothelial lacunae [11]. In physiological conditions, lymphatic flow amounts to $3 \mu \mathrm{L} \cdot \mathrm{min}^{-1} \cdot \mathrm{mm}^{-2}$, flow velocity in initial lymphatics is of the order of $2 \mathrm{~mm} \cdot \mathrm{min}^{-1}$ [1]. Under physiological conditions, a minor share of pleural fluid $(<15 \%)$ could be drained through the visceral pleura in animals where the visceral pleura is perfused by pulmonary circulation. In this case there is a potential absorption gradient as the hydraulic pressure in pulmonary capillaries $\left(\sim 10 \mathrm{cmH}_{2} \mathrm{O}\right)$ is lower than the plasma colloid osmotic pressure $\left(\sim 25 \mathrm{cmH}_{2} \mathrm{O}\right)$ [1]. This mechanism is excluded in humans where the visceral pleura is perfused by the systemic circulation [12]. Some data suggest that active water transport operated by mesothelial cells plays a potential role in pleural fluid turnover [13].

The lymphatic conductance $(K l)$ is $>10$ times higher [7] than the filtration coefficient of the parietal pleura $(K f)$. In fact, the latter includes two membranes in series, the capillary endothelium and the parietal mesothelium, which create a considerable high-resistance system for water and plasma proteins [14, 15]. The visceral pleura are essentially excluded from pleural fluid turnover in physiological conditions because, due to its greater thickness, its permeability is $\geqslant 10$ fold lower compared to that of the parietal pleura $[14,15]$.

There is a polarisation of filtration/drainage for pleural fluid due to intrapleural Pliq gradients as shown in figure 4 . Filtration mostly occurs in the less dependent portions of the cavity while drainage mostly occurs on the diaphragmatic and mediastinal

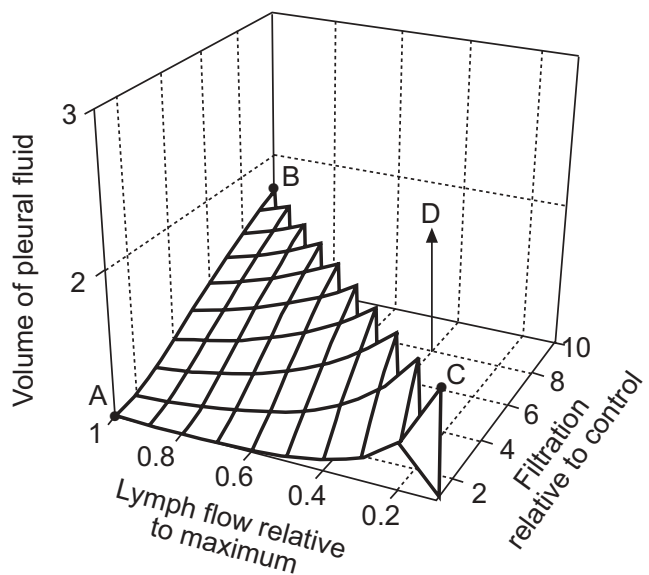

FIGURE 5. Sensitivity analysis describing the features of the lymphatic control on pleural liquid volume. Point A corresponds to the physiological condition. The ordinate shows that pleural liquid volume would only be slightly increased when microvascular filtration is increased 10 times (point B) or when the maximum lymphatic flow is decreased to 1:10 of normal (point $\mathrm{C}$ ). Outside the chequered area (point D) no lymphatic control is operating.

surfaces [1] (as indicated by following radiolabelled markers). Some recirculation of pleural fluid has also been documented $[16,17]$. Pleural liquid volume in physiological conditions averages $\sim 0.2 \mathrm{~mL} \cdot \mathrm{kg}^{-1}$, only $1 \%$ higher than the theoretical minimum corresponding to the case where Pliq $=$ Pabs [7]. Pleural fluid protein concentration averages $\sim 1.5 \mathrm{~g} \cdot \mathrm{dL}^{-1}$ so that the physiological fluid/plasma protein concentration ratio (defined above) averages 0.2 .

\section{Control of pleural fluid volume}

It is certainly difficult to exactly differentiate between the three mechanisms potentially providing pleural fluid transports, namely lymph flow, active water reabsorption and Starling dependent absorption through the visceral pleura. However, it should be stressed that lymphatics, unlike other pathways for fluid removal, act as an efficient negative feedback system to regulate pleural fluid dynamics [7] through two mechanisms: 1) they set a rather sub-atmospheric pressure thanks to their powerful draining action; and 2) they can markedly increase draining flow in response to increased filtration $[7,8]$.

Figure 5 presents a sensitivity analysis depicting the features of the control mechanism of pleural liquid volume based on lymphatic control [7]. The chequered area represents the extent of the control mechanism on pleural liquid volume (the controlled variable), tested by either increasing pleural fluid filtration rate or decreasing the maximum lymphatic draining flow. Point A corresponds to the physiological condition. Point B shows how, for a 10-times increase in filtration rate (due, for example, to an increase in hydraulic permeability), volume of the liquid (Vliq) would not even be doubled due to the increase in lymphatic draining flow. Point $\mathrm{C}$ shows that reducing the maximum lymph flow down to 1:10 of normal (e.g. for mediastinal lymphatic compression or mesothelioma) would still maintain Vliq close to normal, although it would be impossible to match an increase in filtration rate. 
The features of the control mechanisms have been described in an experimental animal model [1, 7]; thus, it appears reasonable to question whether the same mechanism operates in humans. The draining role of parietal lymphatics in humans is demonstrated by the epidemiological finding of the "black spots", namely the accumulation of asbestos fibres around the parietal pleural lymphatic stomata [18]. The interpretation of this finding is that intrapleural translocation of ultrafine asbestos fibres reaching the pleural space [19] is flow-driven towards the pleural fluid draining sites; furthermore, the accumulation at the lymphatic entrance is due to the geometric hindrance of anisodiametric asbestos particles.

\section{Pathophysiology of pleural effusion}

When filtration overwhelms fluid drainage, pleural effusion results; point $\mathrm{D}$ in figure 5, falling outside the chequered area, refers to a condition where the control mechanism becomes ineffective because the maximum lymphatic flow cannot match the increase in filtration rate. In this condition pleural effusion will ensue and the increase in pleural fluid volume will purely reflect a new equilibrium between the pressures acting across compartments.

Pleural effusion is favoured by an increase in systemic capillary pressure and an increase in permeability of the parietal/visceral pleura, a strong limitation to draining lymphatic flow. One shall consider that permeability $\times$ capillary surface area ( $K f$ in equation 1$)$ is a multiplicative factor and may increase by one to two orders of magnitudes to account for the increase in filtration rate.

Pleural effusion is classified as exudates when the fluid/serum total protein ratio (TPR) exceeds 0.5 [20-22]. However, this distinction may just result from the definition of a cut-off value. In fact, by plotting the average TPR values from a database of pleural effusions classified as either exudates or transudates [20] versus the corresponding reflection coefficient values (derived as $\sigma \approx 1-\frac{P e v}{P p l}$ ), an obvious inverse correlation is found (fig. 6), meaning that the increase in TPR is the result of progressively more severe damage to the mesothelium that allows more plasma protein (mostly albumin, having a molecular weight of $\sim 70 \mathrm{kDa}$ ) to leak. In transudates, $\sigma$ is $>0.5$ while in exudates $\sigma$ is $<0.5$. The expected experimental variability around the relationship (as suggested by the grey area in fig. 6) reflects the complex interaction of the increase in mesothelial water permeability (either diffused or localised) and the decrease in protein reflection coefficient (also either diffused or localised). However, it appears justified to relate the degree of mesothelial lesion to the pleural fluid absolute concentration of lactic dehydrogenase [20] (indicated as free lactate dehydrogenase (FLDH)). A cut-off for FLDH has been proposed at $163-200 \mathrm{U} \cdot \mathrm{L}^{-1}$ for diagnostic separation between transudates and exudates [20, 23] and that would then correspond to $\sigma \sim 0.5$.

It is now of interest to interpret some clinical observations concerning the occurrence of pleural effusion of various origin. We will do so keeping in mind that, due to the low permeability of the visceral pleura [1], the pleural cavity and the lung extravascular space appear to be rather independent compartments in terms of fluid exchanges in physiological conditions.

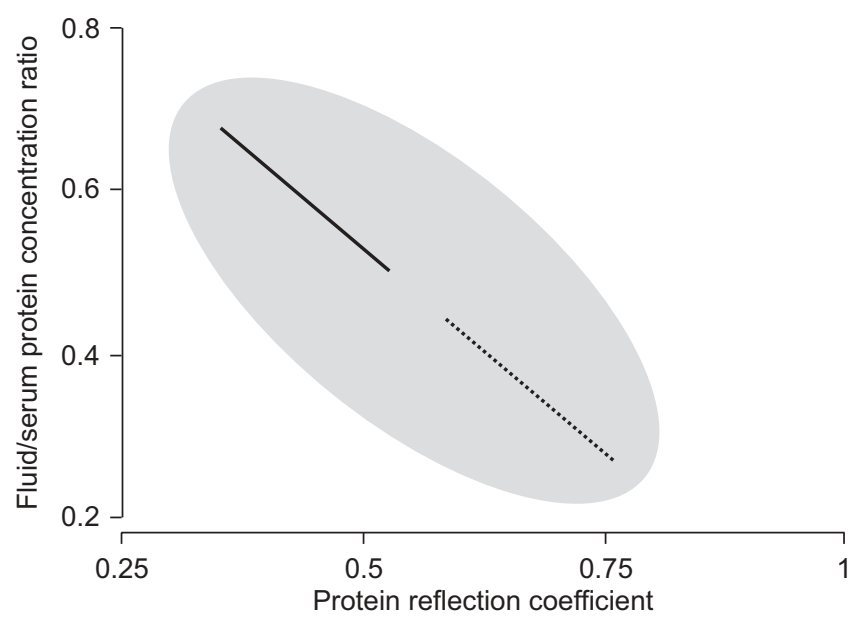

FIGURE 6. Pleural fluid/serum total protein ratio (TPR) (corresponding to $\frac{C e v}{C p l}$ ) plotted versus protein reflection coefficient. The original data were taken from the two regressions presented in [20] referring to exudates and transudates. The figure intends to show that since TPR is a unique function of protein reflection coefficient, moving from transudates $(\cdots \cdots \cdots)$ to exudates (-) just corresponds to progressively increasing damage of the mesothelium.

There is epidemiological evidence that pleural effusion is less frequent in right rather than in left heart failure [24, 25]. These observations are not framed into a specific set of functional parameters (microvascular permeability, lymph flow rate, etc.), therefore, only general considerations can be advanced. The increase in right atrial pressure and central venous pressure should entrain a corresponding increase in filtration rate at capillary level from the parietal pleura. Yet, if pleural effusion is rarely detected even when right atrial pressure exceeds $20 \mathrm{mmHg}$ [25], the interpretation is that the corresponding increase in filtration rate of the parietal pleura can be compensated by lymphatic drainage (fig. 5). The fact that pleural effusion is more frequent in left heart failure (it is present in $\sim 15 \%$ of class IV patients) should be regarded as the result of a marked increase in permeability of the visceral pleura; thus, in this condition pleural cavity and lung extravascular space are no more functionally independent (as they are in the physiological condition). The critical importance of increased permeability in causing pleural effusion is shared by KROEGEL and ANTONY [26]. The combined action of hypoxia and inflammatory state can contribute to the increase in permeability. Furthermore, interstitial lung oedema, by generating positive interstitial pressure, may favour fluid filtration into the pleural cavity. Two more factors may be considered on comparing the visceral with the parietal pleura: the former has an incredibly extended underlying capillary network $\left(2,000 \mathrm{~cm}^{2} \cdot \mathrm{g}^{-1}\right.$ tissue) compared with the endothoracic fascia and has a $\sim 50 \%$ greater surface area due to the interlobar fissures. One shall note that pleural effusion may represent a "physiological finding" during lung water clearance in the first post-natal hours of term newborns [27].

\section{Recovery from pleural effusion}

The full recovery from pleural effusion is a long process mainly due to the fact that the lymphatic mechanism, although being quite adaptive (flow rate can increase by 20 to 30 times), might not match the potential increase in filtration rate 
resulting from the damage of the mesothelium. Data indicate that recovery ranges from weeks (for post-myocardial infarction and post-coronary artery by pass) to months (for tuberculosis and asbestosis) [28]. A decrease in pleural fluid volume can only occur when recovery from inflammation brings the filtration/drainage ratio below unity. This is critically dependent, in turn, upon a restoring a low mesothelial (visceral/parietal) permeability. The Starling mechanism could provide, in principle, fluid reabsorption in the initial phase, although the pressure gradient to sustain it remains quite poor because of the high compliance of the pleural space. Lymphatics will progressively contribute to liquid drainage at a later phase when the Starling dependent absorption is waning on progressively decreasing pleural liquid pressure.

\section{LUNG INTERSTITIAL FLUID}

The lung is a very efficient diffuser due to the enormous surface area of the air-blood barrier. Most of the diffusion occurs in the so called "thin" portion that accounts for almost $50 \%$ of the barrier surface and whose thinness is as low as 0.2-0.3 $\mu \mathrm{m}$ [29]. The "thin portion" is made of the endothelium, the epithelium and an intervening fused basement membrane. The fibrillar molecular component, mostly present in the "thick portion" of the air-blood barrier, includes collagen I and III and elastic fibers whose main role is to provide the elasticity of the lung tissue. The other important molecular component includes the proteoglycan family. The heparan sulphate sub-family (300$500 \mathrm{kDa}$ ) is in the basement membrane, while the chondroitin sulphate sub-family ( $>1000 \mathrm{kDa})$ is bound to hyaluronan in the rest of the matrix [30]. These molecules control the permeability of the pores or channels through which water circulates, particularly at the level of the basement membrane, through their steric hindrance. They also act as link proteins through low-energy ionic and/or hydrophobic non-covalent linkages with other molecules and cells. Such linking action has two important mechanical effects: it allows reciprocal movements of the structures but, at the same time, it provides rigidity to the lung interstitium. Proteoglycans also play a pivotal role in tissue development and repair by interacting with inflammatory cells, proteases and growth factors [30].

\section{Steady state fluid dynamics in the lung interstitial space}

The diffusional fluxes of oxygen across the capillary wall are of the order of $10^{-2} \mathrm{~mL} \cdot \mathrm{cm}^{-2}$ per day, while capillary filtration is as low as $4 \times 10^{-8} \mathrm{~mL} \cdot \mathrm{cm}^{-2}$ within the same time, i.e. six orders of magnitude lower. Both fluxes occur through an incredible development of the capillary network $\left(\sim 2,000 \mathrm{~cm}^{2} \cdot \mathrm{g}^{-1}\right.$ lung tissue). The thinness of the air-blood barrier reflects a functionally "dry" condition, therefore, as much as for the pleural liquid. In addition, for the extravascular space of the lung one can speak of a "minimum" volume of water. In fact, when fluid fluxes increase due to alteration in fluid dynamics, this results in an impairment of diffusion.

The net filtration pressure gradient across the capillary endothelium is largely dominated by the rather sub-atmospheric interstitial pressure that reflects the equilibrium between the capillary filtration through the low-permeability endothelial barrier and the strong draining action of lymphatics. This sub-atmospheric pressure was measured by developing a technique to allow measurements in lungs normally expanded in the chest wall [3], thus maintaining the force vectors acting on the extravascular fluid as shown in figure 2. An increase in elastic forces (over inflation) is a potent factor rendering Pi more sub-atmospheric [31]; a similar result would be caused by a decrease in alveolar surface tension favouring lung collapse.

\section{Control of lung interstitial water}

The lung is strongly resistant to the development of severe oedema. In fact, several mechanisms cooperate to control the volume of the extravascular fluid and to allow minimal variations relative to the steady state value when conditions of increased microvascular filtration are set, a crucial point to guarantee gas diffusion. A key role in buffering the increase in extravascular water is played by proteoglycans in two ways. First, their glycosaminoglycan chains can bind excess water in the interstitial space to form gel-like structures; in turn, the hydration level increases the steric hindrance of proteoglycans and decreases permeability, particularly at the level of the basement membrane. The other important role played by proteoglycans reflects their macromolecular assembly that assures low tissue compliance. In fact, as shown in figure 7 , in response to increased filtration, a minor increase in extravascular water $(<10 \%)$ causes an increase in $\mathrm{Pi}$ by $\sim 15 \mathrm{cmH}_{2} \mathrm{O}$ (from approximately -10 to $5 \mathrm{cmH}_{2} \mathrm{O}$ ) [9]. The significance of this mechanical feature is clear: the marked increase in $\mathrm{Pi}$ buffers further filtration (so called "tissue safety factor") and, at the same time, it increases lymphatic flow (equations 2 and 4). Fluid accumulation occurs mainly in the thick portion of the air-blood barrier where the proteoglycan mesh is not as tight [29], thus avoiding thickening of the thin portion. Another potential factor contributing to control of lung water is the marked pre-capillary arteriolar resistance that contributes to keeping the capillary pressure at the physiological level of $10 \mathrm{cmH}_{2} \mathrm{O}[32,33]$.

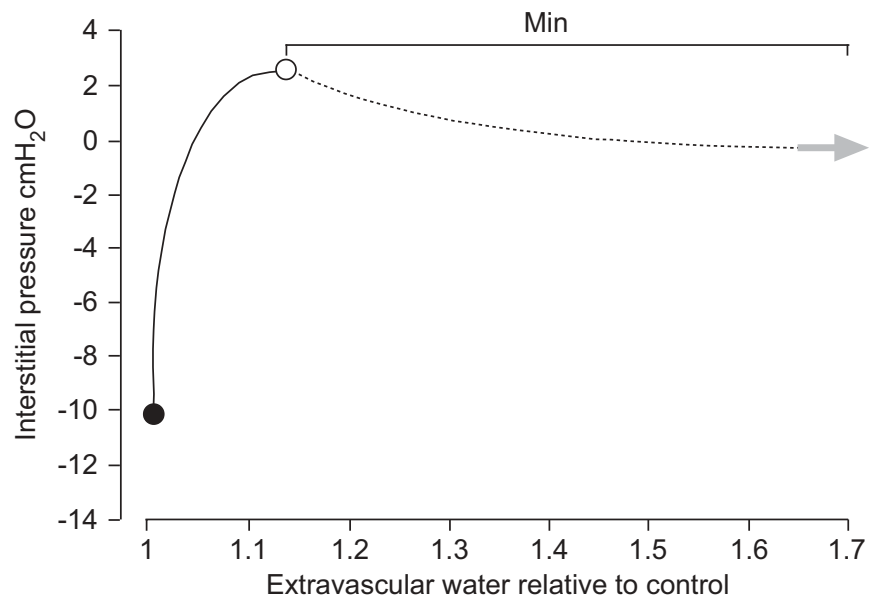

FIGURE 7. The continuous line shows the time course of lung interstitial pressure when interstitial oedema develops $(\bigcirc)$. Note the marked increase in interstitia pressure for a minor change in extravascular water reflecting the very low compliance of the lung interstitial matrix. The dotted line shows the change in interstitial pressure when severe oedema develops (grey arrow). The decrease in pressure reflects the loss of integrity of the macromolecular structure of the extracellular matrix due to fragmentation of proteoglycans, which results in an increase in tissue compliance and in microvascular permeability. Restoring the filtration gradient leads to unopposed filtration and severe oedema (arrow). • control. 


\section{Pathophysiology of severe lung oedema}

The experimental evidence is that the lung can withstand for a long time in a condition of interstitial oedema but the transition to severe oedema is a tumultuous event developing within minutes [9]. We identified the sequence of events leading to the increase in extravascular lung water [9]. A sustained condition of interstitial oedema indeed results in loss of integrity of the native architecture of the large proteoglycans of the interstitial matrix and also of the intermediate sized proteoglycans of the basement membrane [9]. Therefore, as a result of fragmentation, two important features are lost, namely the high tissue elastance and the low permeability of the capillary endothelium. A combination of these two effects leads to the "accelerated phase" of development of severe lung oedema. More specifically, the increase in tissue compliance leads to a decrease in $\mathrm{Pi}$ (fig. 7), thus, restoring a transendothelial Starling pressure gradient. However, although this gradient is smaller than that occurring in physiological conditions, it causes an unopposed increase in microvascular filtration due to the increase in microvascular permeability. In the so called "hydraulic oedema" induced by venous fluid overloading, the degradation process initially involves large matrix proteoglycans, while in the lesional type of oedema (as in acute pancreatitits) there is a major initial degradation of proteoglycans of the basement membrane. Thus, there may be a variable contribution to oedema formation due to the loss of "tissue safety factor" and/or to the increase in microvascular permeability. Despite these differences, we provide a common pathophysiological interpretation for the development of severe oedema as this occurs when a critical threshold is overcome in the process of proteoglycan fragmentation [9]. Factors contributing to progressive disorganisation/fragmentation of the proteoglycan mesh include: 1) mechanical yielding; 2) weakening of the noncovalent bonds of proteoglycans with other matrix components at link sites due to increased hydration/distance; and 3) the activation of tissue matrix metalloproteases (MMP)2 and MMP9, as is specifically the case for hypoxia exposure [34].

The increase in permeability leading to alveolar flooding may occur through porosity of the paracellular pathway of the order of 50-100 nm, which easily allows passage of albumin. Red blood cells can also be found in the alveolar fluid and this reflects major lesion of the air-blood barrier.

It is of interest to discuss the model of control of extravascular lung fluid considering the case of the transplanted lung which, of course, is deprived of lymphatics, whose regeneration might require $\sim 3$ weeks [35]. Pulmonary oedema is reported in $\sim 55 \%$ of the cases [36]; however, in a good percentage of cases, the transplanted lung can act as a good diffuser essentially revealing that the state of the extracellular matrix can assure a tissue safety factor. The major complication within the first 24 $48 \mathrm{~h}$ following transplantation is severe oedema ( $\sim 10 \%$ of the cases) attributed to ischemia-reperfusion injury [37]. A good body of literature focuses on the central role of neutrophil activation [37], which induces reactive oxygen species causing a major increase in microvascular permeability, diffuse alveolar damage and inhibition of the active alveolar fluid reabsorption [38]. Fragmentation of extracellular matrix and lack of clearance of the fragments is involved in neutrophil activation [39]. Lung preservation and reperfusion techniques were in fact revised in order to prevent ischemia-reperfusion injury [40].

Several factors may interact to influence the severity of lung oedema (all in equation 1). The most potent factor is the increase in $K f$ (capillary surface area $\times$ permeability, equation 1). All conditions leading to an increase in cardiac output cause an increase in capillary surface area due to capillary recruitment, such as hypoxia in strenuous exercise. Left heart failure also causes an increase in capillary surface area. Hypoxia [34, 41], inflammatory states [42] and increase in shear rate at capillary level [43] also cause an increase in permeability. Shear rate increases when flow velocity increases with an increase in cardiac output (such as in hypoxia/exercise) or a decrease in vascular bed (such as after lobectomy). The other factor involved in oedema formation is the increase in capillary pressure above the physiological value of $\sim 10 \mathrm{cmH}_{2} \mathrm{O}$. This is considered to occur as a complication of pulmonary hypertension, the hypothesis being that sudden release of the arteriolar vasomotor tone might locally induce an increase in capillary pressure.

\section{Recovery from lung oedema}

Unlike pleural effusion, the recovery from lung oedema may be a question of hours. This requires redeposition and remodelling of the extracellular matrix, in particular as far as the proteoglycan family is concerned. A key, still unsolved, issue is the understanding of how the cell-tissue interaction influences either the reparative process or the development of a severe form of lung oedema such as acute respiratory distress syndrome. Thus, it might be useful to identify interstitial oedema as a sharp edge between tissue repair or manifestation of a severe disease. There is evidence that removal of matrix fragments is critical for successful repair [44]; in particular failure to clear hyaluronan fragments leads to "unremitting" inflammation [45].

We explored the cellular-tissue mechanical interaction evoked by the increase in parenchymal stresses. Interstitial lung oedema was found to cause a considerable increase of mRNA transcripts for tumour necrosis factor- $\alpha$, interleukin- 6 and fibroblast growth factor- $\beta$ [46]. Tissue remodelling has already been described in the phase of interstitial lung oedema and is considered to be a result of the signalling-transduction processes initiated by endothelial and epithelial cells. In fact, signs of activation of these cells corresponding to the phase of increased $\mathrm{Pi}$ were demonstrated by differential expression of lipid microdomains, either caveolae or lipid rafts, which represent specialised signalling-transduction platforms on the plasma membrane. The expression of caveolae increased substantially in response to cardiogenic lung oedema [47-49] which causes major initial damage to large matrix proteoglycans. However, the expression of lipid rafts was increased in response to hypoxic oedema [50], which induces major initial fragmentation of heparansulphate proteoglycans of the basement membrane. Remodelling of the expression of lipid microdomain seems to be a rather fast response based on relocation of available constituents on the plasma membrane surface [51]. It appears tempting to think of these differential activations as fine mechanisms tuned to promote a specific reparative process to resist oedema formation. Endothelial and epithelial cells may indeed act as good sensors of changes in 
force/pressure applied on their surface to elicit a signallingmechano-transduction response [49]. In fact, they are highly deformed in situ due to attachments to adjacent cells and to the basement membrane. Thus, because of these physical constraints they act as rigid structures, an important feature for mechano-transduction [49].

\section{Early diagnosis of lung oedema}

As mentioned previously, interstitial oedema represents a potential unstable condition between tissue repair and severe tissue lesion and, in clinical practice, it is still critical to detect markers of impending risk of developing severe oedema. Based on the notion that interstitial oedema increases the parenchymal stresses, we monitored the changes in lung mechanics induced by an experimental model of lung oedema by forced oscillation technique. We found that reactance measured at a low frequency $(4-5 \mathrm{~Hz})$ decreased progressively and significantly over time when interstitial oedema was present and water accumulation in the interstitial compartment was steadily maintained within $10 \%$ of control value [52] No change in small airways patency was demonstrated so that the finding could be attributed to changes in mechanical properties of the lung tissue under conditions of increased parenchymal stresses. It was concluded that monitoring of reactance could have a potential impact in clinical practice as an early marker of developing frank oedema, before any change in lung compliance or alveolar fluid accumulation occurs.

\section{CONCLUSION}

The common feature of the control mechanism of pleural fluid and interstitial lung water is that, in both compartments, the control relies on an adequate increase of lymph flow to match the increase in filtration rate and such increase is determined by the increase in liquid pressure which, in turn, depends upon the compliance of the compartment. These control mechanisms are quite powerful but display differences and similarities that can be discussed in terms of "gain", "operational features" and "efficiency" of the lymphatic control.

We first discuss the gain, given by $\frac{\Delta J l}{\Delta P e v}=K l$, by answering the question: how much does lymph flow increase for a unit increase in liquid pressure? For the pleural cavity the gain is high. $\mathrm{Kl}\left(\sim 0.8 \mathrm{~mL} \cdot \mathrm{h}^{-1} \cdot \mathrm{cmH}_{2} \mathrm{O}^{-1}\right.$, a high value reflecting the extension of the lymphatic network) should in fact match an increase in filtration rate through a surface area estimated at $\sim 0.2 \mathrm{~m}^{2}$ (including both the parietal and the visceral pleura) causing a small change in Pliq due to high specific compliance of the pleural cavity $\left(\sim 10 \mathrm{cmH}_{2} \mathrm{O}^{-1}\right)$. Now let us consider the gain for the lung interstitium. First, note that the overall filtration surface area through the capillary walls is of the order of $100 \mathrm{~m}^{2}$ (50 times larger than the pleural surface) and, although $K l$ has not been estimated for the lung, it appears difficult to envisage an extension of the lymphatic network proportional to the overall filtration surface area simply because of anatomical constraint imposed by the thinness of the air-blood barrier. Therefore, $K l$ of lung interstitium is probably $<<K l$ of pleural space and, therefore, the gain of interstitial lymphatics is smaller than the gain of pleural lymphatics. Also consider that the change in lung interstitial pressure following an increase in filtration rate is much higher due to the low specific compliance of lung interstitium. Therefore, $K l$ in the lung does not need to be very high as long as the low-tissue compliance represents an efficient mean to counteract filtration.

Now let us consider the operational features as given by the $\frac{K l}{C^{\prime}}$ ratio. For the pleural space, both the numerator and the denominator are high values, while for the lung interstitium both terms are lower so that, as a matter of fact, the ratio might not be too different in the two compartments. Since $\frac{K l}{C^{\prime}}=\frac{\Delta J l}{\Delta V^{\prime}}$, it follows that the efficiency of the lymphatic control, namely $\frac{\Delta J l}{\Delta V^{\prime}}$, is also similar. This can be grasped by answering the following question: how much should lymph flow increase for a unit increase in liquid volume? Bearing in mind that the increase in lymph flow should match the increase in filtration rate, one can note that: 1) for the pleural space, lymph flow should increase a lot for a minor increase in pleural liquid volume because this corresponds to a consistent increase in filtration rate, thus efficiency is quite high; and 2) for the lung interstitium the increase in lymph flow just has to match the increase in extravascular volume which is strongly limited by the low tissue compliance, thus, again, efficiency is high.

In summary, the volume of the pleural fluid and of the lung interstitial compartment are in fact so strictly controlled that it is difficult to detect initial deviations from the physiological state. In practice, a great advantage in the physiological setting turns to be a disadvantage on a clinical basis as it prevents an early diagnosis of developing disease. These considerations highlight the need to find diagnostic tools sensitive enough for early diagnosis.

\section{STATEMENT OF INTEREST}

G. Miserocchi obtained research funds from the Minister of Education in Italy and used them for basic research studies with no personal profit.

\section{REFERENCES}

1 Miserocchi G, Negrini D. Pleural space: pressures and fluid dynamics. In: Crystal RG, West JB, Weibel ER, Barnes PJ, eds. The Lung: Scientific Foundations. Volume 1. New York, Raven Press, 1997; pp. 1217-1225.

2 Hills BA. Graphite-like lubrication of mesothelium by oligolamellar pleural surfactant. J Appl Physiol 1992; 73: 1034-1039.

3 Miserocchi G, Negrini D, Gonano C. Direct measurements of interstitial pulmonary pressure in in-situ lung with intact pleural space. J Appl Physiol 1990; 69: 2168-2174.

4 Levick JR. Capillary filtration-absorption balance reconsidered in light of dynamic extravascular factors. Exper Physiol 1991; 76: 825-857.

5 Michel CC, Curry FE. Microvascular permeability. Physiol Rev 1999; 79: 703-761.

6 Miserocchi G, Negrini D, Gonano C, et al. Liquid drainage through the peritoneal diaphragmatic surface. J Appl Physiol 1989; 66: 1579-1585.

7 Miserocchi G, Venturoli D, Negrini D, et al. Model of pleural fluid turnover. J Appl Physiol 1993; 75: 1798-1806.

8 Miserocchi G. Physiology and pathophysiology of pleural fluid turnover. Eur Respir J 1997; 10: 219-225.

9 Miserocchi G, Negrini D, Passi A, et al. Development of lung edema: interstitial fluid dynamics and molecular structure. News Physiol Sci 2001; 16: 66-71.

10 Wang NS. Anatomy of the pleura. Clin Chest Med 1998; 19: 229-240. 
11 Negrini D, Del Fabbro M, Gonano C, et al. Distribution of diaphragmatic lacunae. J Appl Physiol 1992; 72: 1166-1172.

12 Bernaudin JF, Fleury J. Anatomy of the blood and lymphatic circulation of the pleural serosa. In: Chrétien J, Bignon J, Hirsch A, eds. The Pleura in Health and Disease. New York, Marcel Dekker Inc., 1985; pp. 101-124.

13 Zocchi L. Physiology and pathophysiology of pleural fluid turnover. Eur Respir J 2002; 20: 1548-1558.

14 Negrini D, Reed RK, Miserocchi G. Permeabiliry-surface area product and reflection coefficient of the parietal pleura in dogs. $J$ Appl Physiol 1991; 71: 2543-2547.

15 Negrini D, Townseley MI, Taylor AE. Hydraulic conductivity and osmotic reflection coefficient of canine parietal pleura in vivo. J Appl Physiol 1994; 76: 627-633.

16 Butler JP, Huang J, Loring SH, et al. Model for a pump that drives circulation of pleural fluid. J Appl Physiol 1995; 78: 23-29.

17 Haber R, Grotberg JB, Glucksberg MR, et al. Steady-state pleural fluid flow and pressure and the effects of lung buoyancy. J Biomech Engin 2001; 124: 485-492.

18 Boutin C, Dumortier P, Rey F, et al. Blackspots concentrate oncogenic asbestos fibres in the parietal pleura. Am J Respir Crit Care 1996; 153: 444-449.

19 Miserocchi G, Sancini G, Mantegazza F, et al. Translocation pathways for inhaled asbestos fibers. Environ Health 2008; 7: 4.

20 Joseph J, Badrinath P, Basran GS, et al. Is the pleural fluid transudate or exudate? A revisit of the diagnostic criteria. Thorax 2001; 56: 867-870.

21 Joseph J, Badrinath P, Basran GS, et al. Is albumin gradient or fluid to serum albumin ratio better than the pleural fluid lactate dehydroginase in the diagnostic of separation of pleural effusion? BMC Pulmonary Medicine 2002; 2: 1.

22 Joseph J, Badrinath P, Basran GS, et al. Do we need all three criteria for the diagnostic separation of pleural fluid into transudates and exudates? An appraisal of the traditional criteria. Med Sci Monit 2003; 9: CR474-CR476.

23 Light RW, Macgregor MI, Luchsinger PC, et al. Pleural effusions: the diagnostic separation of transudates and exudates. Ann Intern Med 1972; 77: 507-513.

24 Wiener-Kronish JP, Matthay RA, Biondi JW, et al. Lack of association of pleural effusion with chronic pulmonary arterial and right atrial hypertension. Chest 1987; 92: 967-970.

25 Gao K, Xue P, Zhang Y, et al. Potential role of human visceral pleura in pleural liquid turnover. Clin Med J 2006; 119: 250-254.

26 Kroegel C, Antony VB. Immunobiology of pleural inflammation: potential implications for pathogenesis, diagnosis and therapy. Eur Respir J 1997; 10: 2411-2418.

27 Miserocchi G, Haxhiu Poskurica B, Del Fabbro M. Pulmonary interstitial pressure in anesthetized, paralyzed newborn rabbits. J Appl Physiol 1994; 77: 2260-2268.

28 Cohen M, Sahn SA. Resolution of pleural effusions. Chest 2001; 119: 1547-1562.

29 Conforti E, Fenoglio C, Bernocchi G, et al. Morpho-functional analysis of lung tissue in mild interstitial edema. Am J Physiol Lung Cell Mol Physiol 2002; 282: L766-L774.

30 Hascall V, Hascall G. Proteoglycans. In: Hay ED, ed. Cell Biology of Extracellular Matrix. Orlando, Academic Press Publisher, 1994; pp. 390-417.

31 Miserocchi G, Negrini D, Gonano C. Parenchymal stress affects interstitial and pleural pressures in in situ lung. J Appl Physiol 1991; 71: 1967-1972.
32 Negrini D. Pulmonary microvascular pressure profile during development of hydrostatic edema. Microcirculation 1995; 2: 173-180.

33 Miserocchi G. Lung interstitial pressure and structure in acute hypoxia. In: Roach RC, Wagner PD, Hackett PH, eds. Hypoxia and the Circulation. New York, Springer, 2007; pp. 141-157.

34 Miserocchi G, Passi A, Negrini D, et al. Pulmonary interstitial pressure and tissue matrix structure in acute hypoxia. Am J Physiol Lung Cell Mol Physiol 2001; 280: L881-L887.

35 Kline IK, Thomas PA. Canine lung allograft lymphatic alterations. Ann Thorac Surg 1976; 21: 532-535.

36 Khan SU, Salloum J, O Donovan PB, et al. Acute pulmonary edema after lung transplantation. Chest 1999; 116: 187-194.

37 Maurer JR. The lung following transplantation. In: Crystal RG, West JB, Weibel ER, Barnes PJ, eds. The Lung: Scientific Foundations. Volume 2. New York, Raven Press, 1997; pp. 2771-2785.

38 Khimenko PL, Barnard JW, Moore TM, et al. Vascular permeability and epithelial transport effects on lung edema formation in ischemia and reperfusion. J Appl Physiol 1994; 77: 1116-1121.

39 Adair-Kirk TL, Senior RM. Fragments of extracellular matrix as mediators of inflammation. Int J Biochem Cell Biol 2008; 40: 1101-1110.

40 Trocha SD, Kevil CG, Mancini MC, et al. Organ preservation solutions increase endothelial permeability and promote loss of junctional proteins. Ann of Surg 1999; 230: 105-113.

41 Hansen J, Olsen N, Feldt-Rasmussen B, et al. Albuminuria and overall capillary permeability of albumin in acute altitude hypoxia. J Appl Physiol 1994; 76: 1922-1927.

42 Frank PG, Lisanti MP. ICAM-1: role in inflammation and in the regulation of vascular permeability. Am J Physiol Heart Circ Physiol 2008; 295: H926-H927.

43 Min-ho K, Harris NR, Tarbell JM. Regulation of capillary hydraulic conductivity in response to an acute change in shear. Am J Physiol Heart Circ Physiol 2005; 289: H2126-H2135.

44 Teder P, Vandivier RV, Jang D, et al. Resolution of lung inflammation by CD44. Science 2002; 296: 155-158.

45 Noble PW, Jiang D. Matrix regulation of lung injury, inflammation, and repair: the role of innate immunity. Proc Am Thorac Soc 2006; 3: 401-404.

46 Sabbadini M, Barisani D, Conforti E, et al. Gene expression analysis in interstitial lung edema induced by saline infusion. Biochim Biophys Acta 2003; 1638: 149-156.

47 Palestini P, Calvi C, Conforti E, et al. Composition, biophysical properties and morphometry of plasma membranes in pulmonary interstitial edema. Am J Physiol Lung Cell Mol Physiol 2002; 282: L1382-L1390.

48 Palestini $\mathrm{P}$, Calvi C, Conforti E, et al. Compositional changes in lipid microdomains of air-blood barrier plasma membranes in pulmonary interstitial edema. J Appl Physiol 2003; 95: 1446-1452.

49 Daffara R, Botto L, Beretta E, et al. Endothelial cells as early sensors of pulmonary interstitial edema. J Appl Physiol 2004; 97: 1575-1583.

50 Botto L, Beretta E, Daffara R, et al. Biochemical and morphological changes in endothelial cells in response to hypoxic interstitial edema. Respir Res 2006; 7: 7.

51 Botto L, Beretta E, Bulbarelli A, Rivolta et al. Hypoxia-induced modifications in plasma membranes and lipid microdomains in A549 cells and primary human alveolar cells. J Cell Biochem 2008; 105: 503-513.

52 Dellacà RL, Zannin E, Sancini G, et al. Changes in the mechanical properties of the respiratory system during the development of interstitial lung edema. Respir Res 2008; 9: 51. 